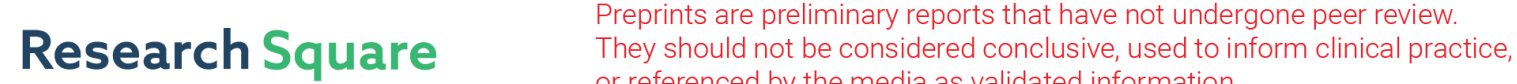 or referenced by the media as validated information. \\ Predictive and Regenerative Medicine for Humans
}

\section{Shun Shun ( $\nabla$ carson1114@hkbn.net)}

Former University of Hong Kong postgraduate student https://orcid.org/0000-0002-0576-5999

\section{Article}

Keywords: Topology and DNA projection, Bayesian prediction methodology, Genome Editing, Spatial Analysis

Posted Date: August 4th, 2020

DOI: https://doi.org/10.21203/rs.3.rs-48726/v1

License: (c) (1) This work is licensed under a Creative Commons Attribution 4.0 International License. Read Full License

Version of Record: A version of this preprint was published at Asian Journal of Mathematical Sciences on December 1st, 2021. See the published version at https://doi.org/10.22377/ajmsmds. 


\title{
Predictive and Regenerative Medicine for Humans
}

\author{
Abstract
}

In human history, finding a cure for the devastating disease of cancer has been a long, ongoing battle. Recently, scientific research has shown several feasible bio-medicinal ways for the treatment of cancer. The first method is through predictive regenerative medicine, which converts (breast) cancer cells back into phenotypically benign cells ${ }^{1}$ using continually updated predictions from Bayesian inference models ${ }^{2}$. Another common method is the rewriting of damaged coordinating genes, but this has underlying moral issues — who can authorise the right to alter human genes? To investigate these methods, this paper will discuss elementary point-set topologies, physical gene mapping, and gene sequencing. The main purpose of this paper is to establish a statistical method with the help of mathematics in our biological life science. The proposed method can be applied in the case of manipulating genetic transcription and expression with a suitable Bayesian inference model (for genetic reprogramming). The peak value of cancer cell conversion is its Bayesian regression (based on the model's parameters) from the corresponding Bayesian inference (for the normal posterior distribution, prior and likelihood). The relevant predictive conditions can be determined from the respective model, which could be used to reveal elements that help cancer cell conversion maximisation/minimisation ${ }^{3}$. Practically, we may apply

\footnotetext{
${ }^{1}$ https://www.ncbi.nlm.nih.gov/pmc/articles/PMC6716904/

${ }^{2}$ https://www.ncbi.nlm.nih.gov/pmc/articles/PMC5930008/

${ }^{3}$ Find out the posterior distribution's model parameter $\rightarrow>$ modelling the conversion of cancer cell to normal cell $->$ discover the peak of conversion $->$ find out those conditions that prevent the conversion $->$ increase the rate of recovering from cancer $->$ achieve the goal: minimise the barrier and maximise those favourable factors for reprogramming
} 
both of the Bayesian optimisation and the related attribute adjustment (to the Oct4, SoX2, Klf4 and c-Myc transcription factors) that can induce the reprogramming of cancer cells. The expected outcome is that we can biomedical-engine the cancer cells conversion. To go ahead a step, one may apply my proposed Net-Seizing Theory to capture the genetic mutations that underly cancer disease. It is hope that we can design the corresponding universal strategy to heal cancer.

Keyword: Topology \& DNA projection, Bayesian prediction methodology, Genome Editing, Spatial Analysis

\section{The Origin of the Regenerative Medicine}

This story originated in ancient Greece, and was introduced by Hesiod (Polykandriotis, 2010) [5] in the $8^{\text {th }}$ century B.C. The story explains that Theogony stole fire and gave it to people 
— representing civilisation and technology. He was then punished by Jupiter, who chained Theogony to the Carpathian Mountain. Adding to his suffering, an eagle named Ethan would pick at his liver every day, yet his liver would regenerate each night (Polykandriotis, 2010) [5]. Finally, he was freed by Hercules after 30,000 years of torture. This story shows that the ancient Greeks were aware of the liver's regenerating capacity, and hence called it "hepar" (Polykandriotis, 2010) [5], which means the liver can "repair itself".

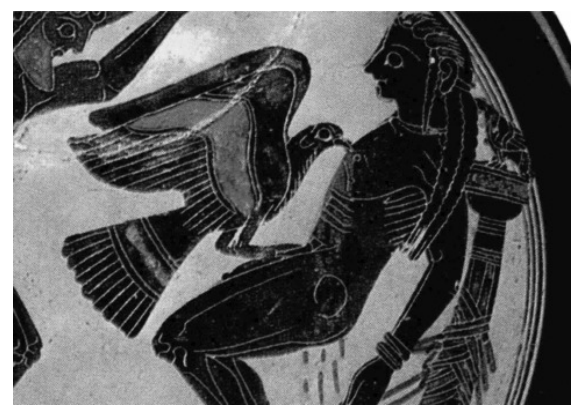

Figure 1. The myth of the Titan Prometheus provides an icon for RegMed (PolyKandriotis, 2010) [5]

Another ancient scripture is Genesis, stating that "the Lord God build up into a woman the rib that he had taken from the man" (Polykandriotis, 2010) [5]. Similar occurrences have happened in modern times, such as tissue replacement demonstrated by brothers, Cosmas and Damian, who transplanted a Moor's leg due to the patient's disease. The earliest detailed research in regenerative medicine was by Aristotle in the following texts: History of Animals, Generation of Animals, and Parts of Animals. In his work, Aristotle found that animals in early developmental stages had a higher potential for regeneration (Polykandriotis, 2010) [5]. Aristotle also described the limb regeneration of salamanders and the antlers of deer. He finally concluded that biological forms originate from undifferentiated matter and later become "epigenesis" (Polykandriotis, 2010) [5]. These stories can be categorized as "modern regeneration", and the concept of regenerative medicine has been documented in ancient manuscripts. The purpose of 
the present article is to investigate both T-cell therapy and cancer cell conversion from the topological and Bayesian Inference viewpoints. Thus, one may advance towards a more general future treatment for cancer.

\section{Mathematical Background - Topologies Theory}

In order to fully understand the concept behind gene mapping, a brief review of topology theories and definitions is necessary. There is a review because the notion of mapping stems from this principle — topology. Topology starts from metric space and extends to topological space. From this, homeomorphism mapping to the path of a fundamental group is possible for path lifting and homotopy in covering spaces.

The following is adopted from Dixmier in 1984 [1]:

1. Metric Space

Let $\mathrm{E}$ be a set. A metric on $\mathrm{E}$ is a function d, defined on $\mathrm{E} \times \mathrm{E}$, with real values greater than or equal to zero, satisfying the following conditions:

(i) $\quad \mathrm{d}(\mathrm{m}, \mathrm{n})=0$ iff $\mathrm{m}=\mathrm{n}$;

(ii) $\mathrm{d}(\mathrm{m}, \mathrm{n})=\mathrm{d}(\mathrm{n}, \mathrm{n}) \forall \mathrm{m}, \mathrm{n} \in \mathrm{E}$;

(iii) $\mathrm{d}(\mathrm{m}, \mathrm{o}) \leq \mathrm{d}(\mathrm{m}, \mathrm{n})+\mathrm{d}(\mathrm{n}, \mathrm{o}) \forall \mathrm{m}, \mathrm{n}, \mathrm{o} \in \mathrm{E}$.

When a set is equipped with a metric, it is then referred to as a metric space.
A. Open Set 
Suppose $\mathrm{E}$ is a metric space. Let $\mathrm{A} \subset \mathrm{E}$ (Wilkins, 1988) [10]. Say the subset A is open

if, for each $\mathrm{x}_{0} \in \mathrm{A}$, there is an $\varepsilon>0$, where $\forall \mathrm{x} \varepsilon$ E satisfying $\mathrm{d}\left(\mathrm{x}_{0}, \mathrm{x}\right)<\varepsilon$ and $\mathrm{x} \varepsilon \mathrm{A}$.

B. Closed Set

Suppose $\mathrm{E}$ be a metric space. Let $\mathrm{B} \subset \mathrm{E}$. The set $\mathrm{B}$ is said to be closed if the subset

E - B is open (Runde, 2008) [6].

2. Topological Spaces (Viro, 2008) [8]

Suppose $\mathrm{Y}$ is a set. Viro in 2008 [8] mentioned that let the collection of subsets $\mathrm{Y}_{1}, \mathrm{Y}_{2}, \ldots, \mathrm{Y}_{\mathrm{n}}$ be $\Omega$ with:

(i) $\quad Y_{1} \cup Y_{2} \cup \ldots \cup Y_{n} \in \Omega$;

(ii) $\quad \mathrm{Y}_{1} \cap \mathrm{Y}_{2} \cap \ldots \cap \mathrm{Y}_{\mathrm{n}} \in \Omega$;

(iii) $\varnothing$ and $\mathrm{Y} \in \Omega$.

Then:

(i) $\quad \Omega$ forms a topological structure (or a topology on $\mathrm{Y}$ );

(ii) $(\mathrm{Y}, \Omega)$ forms a topological space;

(iii) $\mathrm{y} \in \mathrm{Y}$ is a point of this topological space;

(iv) $\mathrm{Y}_{\mathrm{i}} \in \Omega$ are open sets of $(\mathrm{Y}, \Omega)$ for $\mathrm{i}=1,2, \ldots, \mathrm{n}$ 
The above conditions in the definition of topological space are known as the axioms of topological structure. Next, let us talk about the properties of the space:

A topological space $\mathrm{Y}$ is connected when the empty set $\varnothing$ and the entire $\mathrm{Y}$ are the only two open and closed sets. Otherwise, $\mathrm{Y}$ is known as disconnected (Viro, 2008) [8].

What is a partition of a set? It means one needs to construct a cover of this set where it is the connected point-wisely disjointed subsets. With the aforementioned properties for a covering to a set, this event is also known as the partition (Viro, 2008) [8].

\section{Mapping and Topological Algebra}

Topological algebra usually handles the reciprocal action between subject algebra like spaces, groups and other topic topology (Viro, 2008) [8]. The point set that exists in the structures of topological space and the group are different. The key topological properties of the space are actually determined by the group itself (Viro, 2008) [8].

(i) Homeomorphism - Suppose $\mathrm{f}:(\mathrm{M}, M) \rightarrow(\mathrm{N}, N)$ is a mapping between two topological spaces (Waldmann, 2014) [9]:

(a) The function $\mathrm{f}$ is called a homeomorphism when

(i) it is both bijective and continuous and;

(ii) its inverse $\mathrm{f}^{-1}$ is also continuous (Waldmann, 2014) [9];

(b) When there is a homeomorphism $\mathrm{f}:(\mathrm{M}, M) \rightarrow(\mathrm{N}, N)$, the image $(\mathrm{N}, N)$ and its pre-image spaces (M, $M$ ) are known in homeomorphic (Waldmann, 2014) [9];

(c) When the function $\mathrm{f}$ is both injective and 


$$
\mathrm{f}:(\mathrm{M}, M) \rightarrow(\mathrm{f}(\mathrm{M}), N \mid \mathrm{f}(\mathrm{M}))
$$

is a homeomorphism, we called the $\mathrm{f}$ as embedding (Waldmann, 2014) [9].

In such sense, homeomorphism is in the category which is denoted as "Top" of the topological space.

(ii) Category and morphism - One will call it a category, when there is a collection of

a. objects and;

b. morphisms,

where functions or the morphism between objects will preserve the structure inherent (i.e., a structure preserving map) to the objects (such as groups, vector spaces, topological spaces, differentiable manifolds, etc.) or in a symbolic representation $\mathrm{C}(\mathrm{O}, \mathrm{M})$.

(iii) Isomorphism - in category $\mathrm{C}$, one calls two objects a, $\mathrm{b}$ belongs to $\mathrm{Obj}(\mathrm{C})$ isomorphic if there are morphisms $f$ belongs to Morph $(a, b)$ with $f o g=i d_{b}$ and $\mathrm{g}$ o $\mathrm{f}=\mathrm{id}$. This is the correct notion of isomorphisms.

(iv) Topological Group is a set $\mathrm{S}$ consisting of a topological plus a group of structures. In addition, the map

S x S $\rightarrow$ S: (u, v) $\mid \rightarrow$ uv and S $\rightarrow$ S: $\mathrm{u} \mid \rightarrow \mathrm{v}^{-1}$ will still be continuous (Viro, 2008) [8].

(v) A homomorphism in a topological group is also known as a continuous group homomorphism. Suppose there are two topological groups: G and H. When there is 
a map $\mathrm{f}: \mathrm{G} \rightarrow \mathrm{H}$, if $\mathrm{f}$ is continuous at $\mathrm{l}_{\mathrm{G}}$, then $\mathrm{f}$ is continuous. In this case, it is also called a group homomorphism (Viro, 2008) [8].

In a similar situation, one may define the cases for local isomorphism and homeomorphism in topological groups.

(vi) In brief, an invariant of a knot or link is a property that does not change when the knot or link is deformed in space.

(vii) A topological property is a predicate of topological spaces which is invariant under isomorphism of topological spaces and hence under homeomorphism: the socalled homeomorphism invariance. (N.B. In algebra, isomorphisms are usually called just that - isomorphisms, and they refer to homomorphisms which happen to be invertible and whose inverse is itself a homomorphism. In this sense, homeomorphism is also isomorphism.)

\section{Fundamental Group}

Is it possible to deform a continuous map (Viro, et al., 2008) [8]? Firstly, let us determine details of deformation -- path, homotopy and fundamental group etc.

(i) Path - is a topological space Y. It concerns a continuous map t: $\mathrm{I}=[0,1] \longrightarrow \mathrm{Y}$ where $t(0)$ and $t(1)$ correspond to the starting and ending of $t$. Then one may say $\mathrm{t}(0)$ connects with $\mathrm{t}(1)$ through the path s (Viro, et al., 2008) [8]. 
(ii) Homotopy - Suppose there are two continuous maps, s and t from topological spaces $\mathrm{U}$ to $\mathrm{V}$. In addition, let $\mathrm{J}: \mathrm{U} \mathrm{x} \mathrm{I} \rightarrow \mathrm{V}$ be a continuous map with the properties that $\mathrm{J}(\mathrm{u}, 0)=\mathrm{s}(\mathrm{u})$ and $\mathrm{J}(\mathrm{u}, 1)=\mathrm{t}(\mathrm{u})$ for each $\mathrm{u} \in \mathrm{U}$. Then $\mathrm{s}$ and $\mathrm{t}$ are both said to be homotopic. One may find a homotopy $\mathrm{J}$ that exists between $\mathrm{s}$ and $\mathrm{t}$ (Viro, 2008) [8]. Homotopy equivalence occurs when the situation of isomorphism in topological spaces exists, such that the continuous function has an inverse up to homotopy. This is known as the (weak) Homotopy equivalence (Shick, 2007) [7].

(iii) Suppose $\mathrm{x}_{0}$ is a point of topological space $\mathrm{X}$. A path in $\mathrm{X}$ with the initial point and final point at $\mathrm{x}_{0}$ is known as a loop at $\mathrm{x}_{0}$ in $\mathrm{X}$. One may denote this event by:

(a) $\Omega_{1}\left(\mathrm{X}, \mathrm{x}_{0}\right)$ - the set of loops at point $\mathrm{x}_{0}$ in $\mathrm{X}$;

(b) $\pi_{1}\left(X, x_{0}\right)$ - the set of homotopy classes with loops at $\mathrm{x}_{0}$ in $\mathrm{X}$.

Both $\pi_{1}\left(\mathrm{X}, \mathrm{x}_{0}\right)$ and $\Omega_{1}\left(\mathrm{X}, \mathrm{x}_{0}\right)$ have a multiplicative operation. The set $\pi_{1}\left(\mathrm{X}, \mathrm{x}_{0}\right)$ with multiplication is a group. If in addition, $\pi_{1}\left(\mathrm{X}, \mathrm{x}_{0}\right)$ is the fundamental group, it is also called the Poincare group (Viro, 2008) [8].

\section{Covering Spaces}

Suppose Y and D are topological spaces. In addition, let $\mathrm{p}: \mathrm{Y} \rightarrow \mathrm{D}$ be a continuous map. Assume that there is a surjective mapping $\mathrm{p}$, and each point of D has a neighbourhood V. Furthermore, $\mathrm{p}$ has the character that the preimage $\mathrm{p}^{-1}(\mathrm{~V})$ of $\mathrm{V}$ constitutes a disjoint union of open sets $\mathrm{U}_{\alpha}$, and $\mathrm{p}$ homeomorphically maps each $\mathrm{U}_{\alpha}$ onto $\mathrm{V}$. We say that $\mathrm{p}: \mathrm{Y} \rightarrow \mathrm{D}$ is a covering of $\mathrm{D}$, and the space D is called the base of such covering. $\mathrm{Y}$ is the known as the covering space for $\mathrm{D}$ and also 
the total space of the covering. All of the neighbourhoods V with such properties are said to be trivially covered. One may call the above situation as a covering map or covering projection for the map p.

From the above definition, let us consider the punctured plane $\mathbf{R}^{2} \backslash\{0\}$ in the usual subspace topology.

The complex logarithm function maps a Riemann surface to $\mathbf{R}^{2} \backslash\{0\}$ as illustrated below (Shick, 2007) [7]:

As the complex logarithmic function can be deformed and mapped to a Riemann surface, hence the DNA spiral helix can also be divided and mapped in a similar way to a plane surface once the covering spaces condition is verified. The intuitive idea can be further developed for the $3 \mathrm{D}$ space reduction to a $2 \mathrm{D}$ plane space in gene mapping.
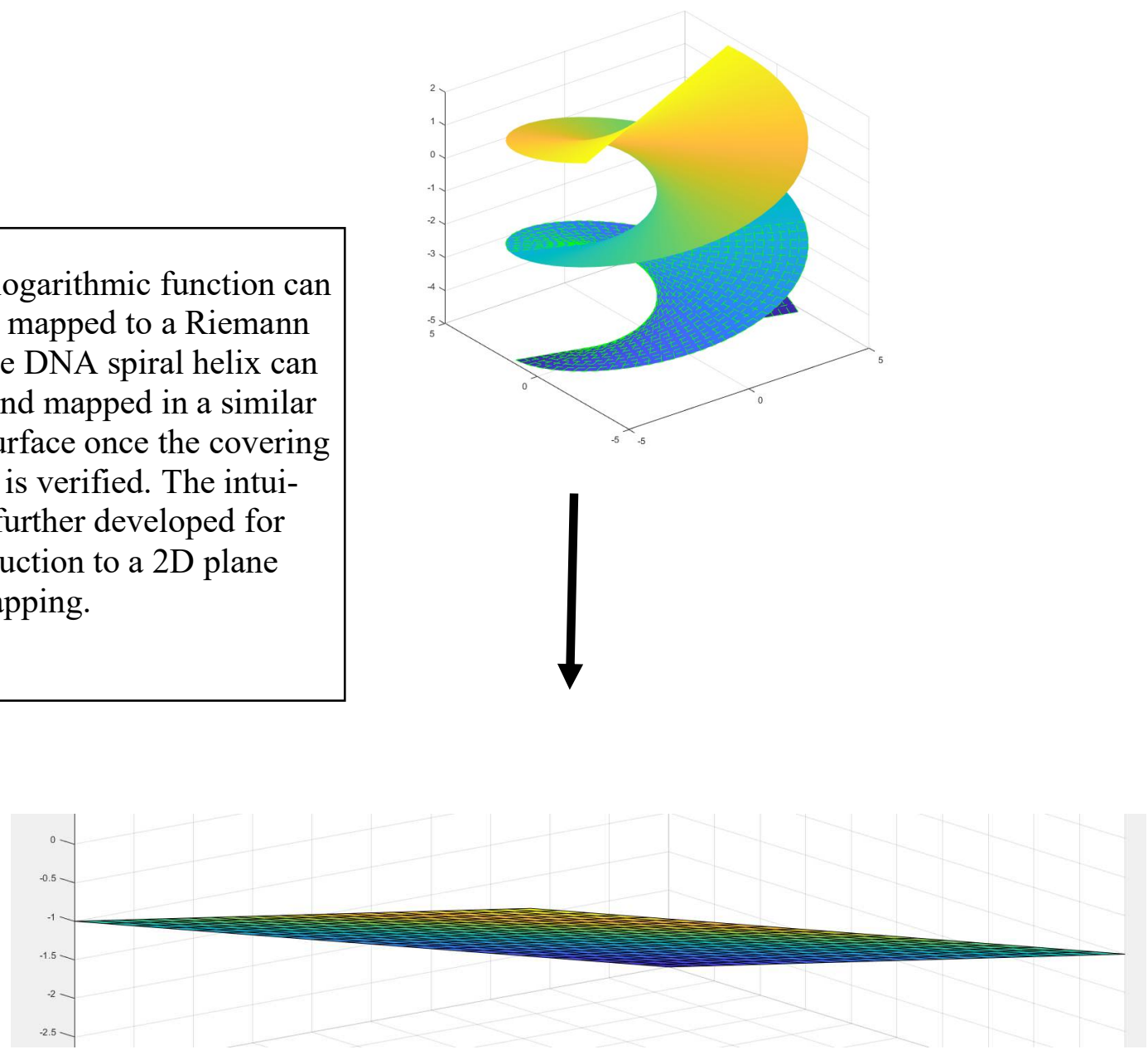
Lifting - Suppose there are arbitrary mappings q: $\mathrm{X} \rightarrow \mathrm{B}$ and $\mathrm{f}: \mathrm{A} \rightarrow \mathrm{B}$. A map g: A $\rightarrow$ X such that $\mathrm{g} \mathrm{o} \mathrm{q}=\mathrm{f}$ is known as to cover $\mathrm{f}$ or be a lift of $\mathrm{f}$ (Viro, 2008) [8]. Actually, the main duty of our lifting problem is to handle those topological problems where one is needed to find a continuous lift of some continuous map (Viro, 2008) [8].

Path Lifting Theorem -- Suppose $\mathrm{q}: \mathrm{X} \rightarrow \mathrm{B}$ is a covering. Suppose further that $\mathrm{x}_{0} \in \mathrm{X}$ and $\mathrm{b}_{0} \in$

B be the points where $\mathrm{q}\left(\mathrm{x}_{0}\right)=\mathrm{b}_{0}$ (Viro, 2008) [8]. Then for any path s: $\mathrm{I} \rightarrow \mathrm{B}$ starting at $\mathrm{b}_{0}$, there is a unique path s': $\mathrm{I} \rightarrow \mathrm{X}$ that also begins at $\mathrm{x}_{0}$ and is a lift of $\mathrm{s}$ or $\mathrm{s}^{\prime}(0)=\mathrm{x}_{0}$ and $\mathrm{s}^{\prime} \mathrm{o} \mathrm{q}=\mathrm{s}$ (Viro, 2008) [8].

Path homotopy property - - for a given covering space $\mathrm{q}: \mathrm{X}^{\prime} \rightarrow \mathrm{X}$, a homotopy $\mathrm{g}_{\mathrm{t}}$ : $\mathrm{Y} \rightarrow \mathrm{X}$ and a map $g_{0}: Y \rightarrow X$ that lifts to $g^{\prime}$, there exists a unique homotopy $g^{\prime}{ }_{t}: Y \rightarrow X^{\prime}$ that lifts $g_{t}^{4}[15]$

\section{Biological Application -- Gene Mapping, Sequencing, and Expression}

From the previous section on mathematical topology theories and definitions, it is clear that biologically, genes can be mapped to the Riemann space. This intuitive idea may finally project the desired DNA from a 3D space and reduce it to a 2D plane space. Then the 2D projected DNA patterns will be deformed and the topological features for DNA are thus obtained. The prescribed scheme depicts the full picture of what topological data analysis is, and eventually the final genome-wide recombination map can be gained. Besides those described above, there are also two types of gene mapping: physical and genetic mapping.

For genetic mapping, the first step is to identify genetic markers and the mapping population. A feasible marker can be achieved by using the special sequence feature that is significantly

\footnotetext{
${ }^{4}$ http://www.math.uchicago.edu/ may/VIGRE/VIGRE2011/REUPapers/Dooley.pdf
} 
different from the parental set. Indeed, the distance between two markers on the chromosome determines the chance of passing on certain characteristics to the next generation. Hence, one may employ "co-segregation" patterns of all markers for the reconstruction of their orders. Therefore, it would be interesting to record the genetic marker with the corresponding genotypes for both parents and their children. The resolution of genetic mapping might become higher as the number of genetic markers and the size of the mapping population increase. Moreover, they can also prevent the mapping from becoming saturated.

Since children have specific genetic marker features, it is possible to distinguish them from their parents. Additionally, their genetic relationship with other genes can be calculated. The computing criteria include genes that have common markers, for which one can identify an actual gene loci and bracketed region that are located between the two most nearby markers. This process can be continued in order to find additional high-resolution markers until the specific causative locus is discovered.

For physical mapping, genomes are first divided into hierarchically smaller pieces. One may then characterize each single piece and reassemble them backwards. The resulting overlapped path of these small pieces would provide details for researchers to investigate the physical distance between discovered genomic features. In fact, the genes can be cut by enzymes or by sonication. After cutting the DNA into fragments, they will be further separated by electrophoresis. Next, a decision can be made regarding the genetic fingerprint of the DNA to be cloned. During fingerprint analysis, contigs will be assembled into overlapping stretches. From this, a choice of clone can be made so that one can find out the DNA sequence for a particular organism.

In essence, genome sequencing requires scientists to break the genome into small pieces, which are then sequenced. Then they attempt to reassemble them in the proper order to finally 
achieve the original sequence of the whole genome. The challenge for researchers is putting together such a giant biological jigsaw puzzle, which can also be seen as a form of decoding.

Gene expression refers to the process of a gene's information being used for the synthesis of a functional gene product. In most instances, the product will be a protein. In some cases, such as non-protein coding genes, the product is functional RNA. In biology, the erasing and remodelling of epigenetic marks, such as in DNA methylation, is known as reprogramming.

One of the most important applications of 3D to 2D mapping is the encouragement of spatial analysis. This process leads to the investigation of population genetics and hence the development of precision (personalised) regenerative medicine for each individual. One can maximise the treatment effect of drugs and minimise their side effects as drugs are always tailormade to suit the individual.

\section{Main Results and Discussions - Relationship between Prior, Posterior, and Likelihood for Bayesian Inference in Immunology}

1. Immunology and Genome Editing

What is genome editing? It is referred to as a type of genetic engineering, where a living organism's DNA can be inserted, deleted, modified, or even replaced. In fact, it is completely different from traditional genetic engineering techniques, in which genetic material can only be randomly added into the gene. The difference is that gene editing can target the insertion to a desired and specified location. Presently, there are four kinds of engineered nucleases. They are known as mega-nucleases, zinc finger nucleases, tran- 
scription activator-like effector-based nucleases (TALEN), and the clustered regularly interspaced short palindromic repeats (CRISPR/Cas9) system ${ }^{5}[16]$. The most recent gene editing method used is the engineered nucleases or the "molecular scissors". The scissors first create genetic site-specific double-strand breaks at the desired location. The breaks are then repaired by the mechanism known as non-homologous end-joining (NHEJ) or homologous recombination $(\mathrm{HR})^{6}[17]$. This results in targeted gene mutation or the gene being "edited". Indeed, ZFNs, CRISPR, and engineered nucleases are all enzyme classes of genome editing. Besides the application in human reproduction, another significant usage is in immunotherapy for cancer treatment.

Immunotherapy is a type of treatment that boosts T-cells in the immune system ${ }^{7}$. T-cells can protect the human body from pathogens and aid in the clearance of mutant cells. These protective actions can be achieved by the specific recognition of T-cell receptors. In the case of cancer immunotherapy, T-cell antitumor efficacy is unleashed from the inhibition of immune checkpoints. In addition, when T-cells are genetically engineered, the adaptive immunity against cancer can be further expanded. This type of cancer immunotherapy has proved to be effective in the case of some hematological malignancies.

\footnotetext{
${ }^{5}$ https://ghr.nlm.nih.gov/primer/genomicresearch/genomeediting

${ }^{6}$ ibid

${ }^{7}$ https://www.cancer.gov/about-cancer/treatment/types/immunotherapy
} 
2. Bayesian Inference and regression for immunology and genome editing

Suppose the probability of a person having cancer and being normal is equal (i.e., 0.5). Assume further that the probability of a patient adopting immunology therapy is 0.9 (adopted) and 0.1 (not adopted). Finally, assume the probability of someone being healed by a chemical conversion drug is 0.7 while the complement is 0.3 . Bayesian inference and Bayesian regression can then be applied to predict the probability that a patient with cancer can be healed by an immunological drug.

The Bayesian tree diagram for the above thought experiment is shown below:

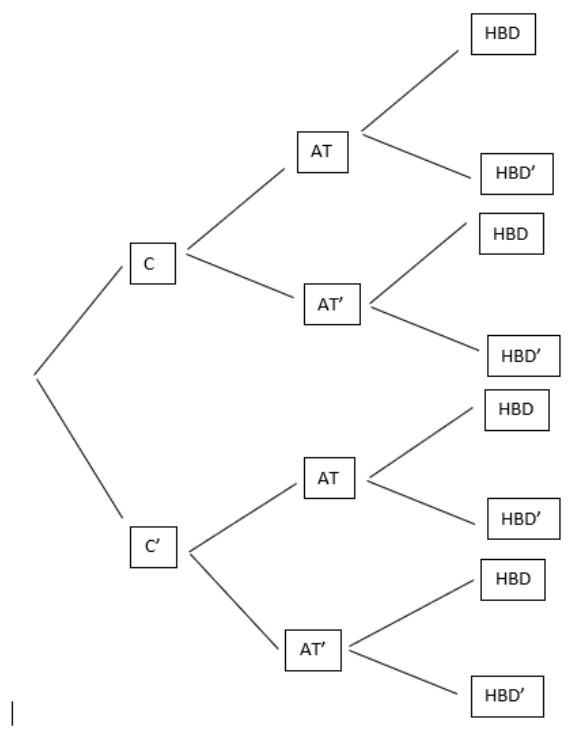

Key: C - cancer

AT - Attain treatment

The following paragraph offers a brief review of Bayesian inference and Bayesian regression.

Of primary interest is the conditional probability:

Pr (Cancer | Healed by the Drug) 
That is the posterior belief of our predicted probability that will be compared with the observation with the evidence, i.e. the number of patients getting cancer ${ }^{8}$ or $\operatorname{Pr}$ (Cancer) [18]. In order to define our model, the likelihood function $\operatorname{Pr}$ (Healed by the Drug | Cancer) and the distribution of prior beliefs is required. The product of these two models provides the posterior belief: $\operatorname{Pr}$ (Cancer | Healed by the Drug) distribution ${ }^{9}$ [19]. Indeed,

$\operatorname{Pr}($ Cancer $\mid$ Healed by the Drug $) \propto \operatorname{Pr}($ Healed by the Drug $\mid$ Cancer $) * \operatorname{Pr}($ Cancer $)$

From the above relationship, it can be shown that: $\operatorname{Pr}($ Cancer $\mid$ Healed by the Drug) will reach its minimum if one can minimize both $\operatorname{Pr}$ (Cancer $\mid$ Healed by the Drug) and the $\operatorname{Pr}$ (Cancer). Alternatively, $\operatorname{Pr}$ (Cancer | Healed by the Drug) will reach its maximum if one can maximize both $\operatorname{Pr}$ (cancer $\mid$ Healed by the Drug) and the Pr (Healed by the Drug $\mid$ Cancer). Cognate situations do apply in the cases of prior belief, posterior belief, and the likelihood of Bayesian inference. This is because the posterior is proportional to [Likelihood * Prior]. Hence, when one fits both likelihood and prior into normal distributions, which is then multiplied, it becomes possible to easily maximize or minimize the posterior belief according to those necessary prescribed requirements. In other words, with knowing both the likelihood and prior belief's distributions, it becomes possible to predict the best posterior belief distribution for further data forecasting and compare the evidenced data from a real situation.

\footnotetext{
${ }^{8}$ https://www.analyticsvidhya.com/blog/2016/06/bayesian-statistics-beginners-simple-english/ ${ }^{9}$ ibid
} 
Next, one needs to consider the following conditional probability matrix A (Pérez-Nimo, et al.,) [4] with two complete sets of events $A_{1}, A_{2}, \ldots A_{n}$ and $B_{1}, B_{2}, \ldots B_{n}{ }^{10}$ [20], one may have:

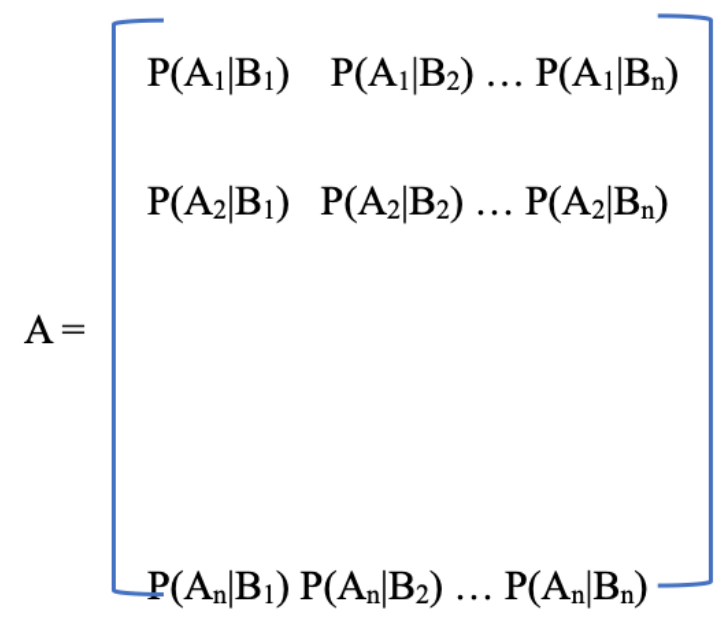

Suppose further that:

$\alpha=\left[\begin{array}{l}\mathrm{P}\left(\mathrm{A}_{1}\right) \\ \mathrm{P}\left(\mathrm{A}_{2}\right) \\ \\ \mathrm{P}\left(\mathrm{A}_{\mathrm{n}}\right)\end{array}\right]\left[\begin{array}{l}\mathrm{P}\left(\mathrm{B}_{1}\right) \\ \mathrm{P}\left(\mathrm{B}_{2}\right) \\ \\ \mathrm{P}\left(\mathrm{B}_{\mathrm{n}}\right)\end{array}\right]$

${ }^{10}$ http://pi.math.cornell.edu/ hatcher/AT/AT-doublepage.pdf 
$A^{*} \beta=\alpha$ (total probability theorem). The Bayesian multivariate linear regression can be applied to obtain the following equation:

$\left[\begin{array}{l}\mathrm{P}\left(\mathrm{A}_{1}, \mu_{1}, \sigma_{1}{ }^{2}\right) \\ \mathrm{P}\left(\mathrm{A}_{2}, \mu_{2}, \sigma_{2}{ }^{2}\right)\end{array}\right]=\left[\begin{array}{ll}\mathrm{x}_{11} & \mathrm{x}_{\mathrm{k} 1} \\ \mathrm{x}_{12} & \mathrm{x}_{\mathrm{k} 2} \\ & \\ & \\ \mathrm{x}_{1 \mathrm{n}}\left(\mathrm{A}_{\mathrm{n}}, \mu_{\mathrm{n}}, \sigma_{\mathrm{n}}{ }^{2}\right)\end{array}\right]\left[\begin{array}{l}\mathrm{x}_{\mathrm{kn} 2} \\ \beta_{1} \\ \beta_{2} \\ \beta_{\mathrm{n}}\end{array}\right]+\left[\begin{array}{l}\varepsilon_{1} \\ \varepsilon_{2} \\ \\ \varepsilon_{\mathrm{n}}\end{array}\right]$

In a similar way, suppose there are other matrix variables $\mathrm{Y}$ and $\mathrm{Z}$ corresponding to...??

When one considers to the series of domino effects caused by cancer, one may obtain:

$$
\mathrm{Y}=\mathrm{XB}+\mathrm{B}_{0} \text { and } \mathrm{Z}=\mathrm{YD}+\mathrm{D}_{0}
$$

then one may have

$$
\mathrm{Z}=\left\{\mathrm{XB}+\mathrm{B}_{0}\right\} \mathrm{D}+\mathrm{D}_{0}
$$

where $\mathrm{XB}+\mathrm{B}_{0}$ and $\mathrm{Z}=\mathrm{YD}+\mathrm{D}_{0}$ is the approximated matrix equation through the linear regression in terms of matrices $\mathrm{X}, \mathrm{Y}$ with $\mathrm{B}_{0}$ and $\mathrm{D}_{0}$ as the error vectors and $\mathrm{B}, \mathrm{D}$ as the corresponding linear transformation vectors. 
Indeed, the actual domino effect in matrix form is:

$\mathrm{A} \rightarrow \mathrm{X} \rightarrow\left[\mathrm{XB}+\mathrm{B}_{0}\right] \rightarrow\left\{\mathrm{XB}+\mathrm{B}_{0}\right\} \mathrm{D}+\mathrm{D}_{0}$

Or in general, $\mathrm{P}(\mathrm{A})$ can be expressed in terms of a Bayesian multiple (with multivariate) regression equation - $(*)$ :

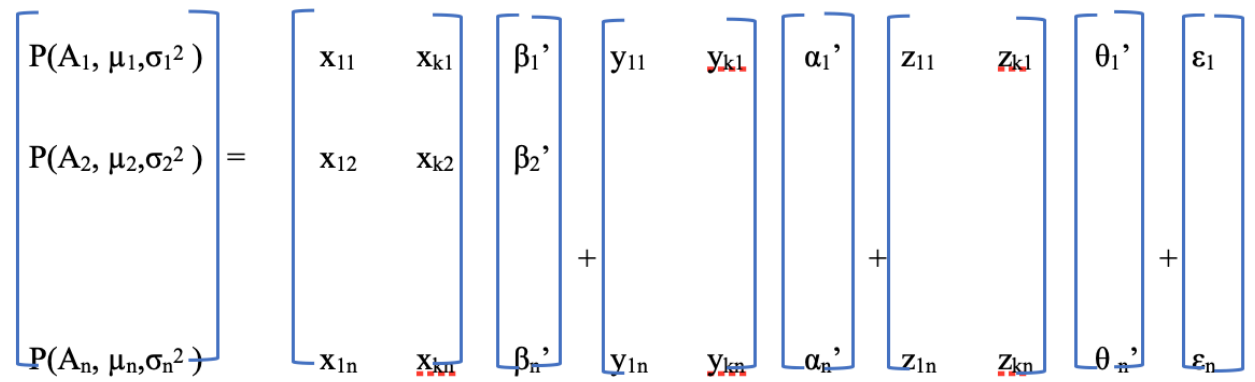

$A=P(A) \beta^{-1}$ where $\beta^{-1}$ is the inverse of the vector $\beta$

Or in terms of matrix notation, one will get the following Bayesian regression equation $(* *)$ from the equation (*) for the conditional probability matrix $\mathbf{A}$ :

$\mathbf{A}=\mathbf{X} \boldsymbol{\beta}^{\prime} \boldsymbol{\beta}^{-1}+\mathbf{Y} \boldsymbol{\alpha}^{\prime} \boldsymbol{\beta}^{-1}+\mathbf{Z} \boldsymbol{\theta}^{\prime} \boldsymbol{\beta}^{-1}+\boldsymbol{\varepsilon}^{\prime}$

where $\mathrm{X}, \mathrm{Y}, \mathrm{Z}$ are matrices in $\left(^{*}\right)$ and $\boldsymbol{\beta}^{\prime}, \boldsymbol{\alpha}^{\prime}, \boldsymbol{\theta}^{\prime}$ are the linear transformation vectors in $\left(^{*}\right)$ and $\boldsymbol{\varepsilon}^{\prime}$ is the error vector of the regression equation $(* *)$.

Practically, for the proposed thought experiment in cancer immunological therapy, it is suggested to substitute those numerical values back into the conditional probability matrix $\mathbf{A}$ and linear transformation vector $\boldsymbol{\beta}$. Then one should find out the corresponding inverse linear transformation vector $\boldsymbol{\beta}^{-1}$ (if it exists) and fit the probability A into a normal distribution with mean $\mu$ and standard deviation $\sigma^{2}$ through Bayesian inference. Thus, an approximate A can be achieved according to the Bayesian regression equation by computing. From this event, a predictive model 
for the regenerative medicine of cancer immunotherapy can be found. In reality, one can run this statistical data through software like SPSS (Statistical Package for the Social Sciences) for a Bayesian Independence test. This helps us find out the prior, likelihood and posterior mean as well as the variance. If one runs this data through software such as JASP (Jeffreys's Amazing Statistics Program), one can find the real predictive model (or an equation) through Bayesian regression. Finally, the connection between matrix A can be evaluated, together with its linear transformation and the corresponding causal relationships for the corresponding human response to cancer drugs (regression analysis plus theory). This also includes a comparison of the data collected for cancer immunotherapy. Indeed, to test how well the Bayesian approximation fits with the error function, we can apply Bayesian optimisation to the chaos connected with the linear regression of the thought cancer drug experiment. All we need to do is computing the probability function of an objective function - the Root Mean Square Error one. Then we can find the gradient descent with the global maximum/minimum. Bayesian optimisation builds a probability model of the objective function by ${ }^{11},{ }^{12}$ :

1. Select several points (samples) from the true RMSE function;

2. Approximate the objective by some other models;

3. Establish the Surrogate model;

4. Go ahead with Gaussian Process model;

5. Obtain the acquisition function;

\footnotetext{
${ }^{11}$ https://towardsdatascience.com/bayesian-optimization-concept-explained-in-layman-terms1d2bcdeaf12f

${ }^{12}$ https://www.r-bloggers.com/bayesian-optimization-of-machine-learning-models/
} 
6. Maximise the acquisition function and get the next approximation point;

7. Repeat the above steps and acquire the best approximated objective function

Furthermore, when there is an attribute adjustment among the transcription factors like Oct4, SoX2, Klf4 and c-Myc, that induces the reprogramming of cancer cells into pluripotent stem cells, we may finally bio-engine our cancer cell conversion ${ }^{13},{ }^{14},{ }^{15}$. To go ahead a step, one may use the snapshot in time to capture the genetic mutations that lay behind the cancer disease ${ }^{16}$. It is no doubt that all cancer is proved to be caused by mutation ${ }^{17}$.

Therefore, I note that the above Bayesian probability distribution (prior, posterior and likelihood) together with the linear regression can form my proposed Net-Seizing theory. The theory tries to catch the Butterfly that lays behind the cancer mutation etc ${ }^{18}$. In fact, my theory consists of the random probability matrix, linear transformation and the causality of domino effects ${ }^{19}$. When the aforementioned computational method (both mathematical and statistical) in the thought experiment well-matches with the real life daily time snapshot research data for the

\footnotetext{
${ }^{13}$ Yuan Jie (2018), Identification of protein kinase inhibitors to reprogram breast cancer cells, Cell Death and disease DOI 10.1038/s41419-018-1002-2

${ }^{14}$ Semi Katsunori (2013), Cellular reprogramming and cancer development, International Journal of Cancer

${ }^{15}$ Lui Beilel (2019), Cancer cell reprogramming: a promising therapy converting malignancy to benignity, BMC, Springer Nature, https://doi.org/10.1186/s40880-019-0393-5

${ }^{16}$ https://hub.jhu.edu/2019/06/28/stem-cell-mutations-affect-disease-risk-over-time/

${ }^{17}$ https://www.hbocsociety.org/how-gene-mutations-cause-cancer.html

${ }^{18}$ https://pubmed.ncbi.nlm.nih.gov/25583473/

${ }^{19}$ When my theory is expressed in terms of both mathematics and statistics, it becomes

[ Random Matrix ] [ LT ] = [Linear regression of the causal domino effects] and mation

[ Linear regression of the causal domino effects $][L T]=[$ Random Matrix $] \quad L T$ is the respective linear transfor-
} 
cancer disease, it is shown that my Net-seizing Theory can be applied in catching the Butterfly

— the chaos and the domino causality of mutations that lay behind cancer. Practically, by identifying those cancer driver genes from the net-seizing, we can develop the corresponding novel drugs and therapies for the disease ${ }^{20}$. Thus, cancer is completely controllable and predictable.

\section{Conclusion - Predictive and Regenerative Medicine}

The significance of this paper is that one may fit the likelihood and prior belief of regenerative medicine with normal distributions (i.e. the method of Bayesian inference). With this, it is possible to forecast the maximum or minimum of posterior beliefs. When this difference is compared with collected data, the researcher can optimize such barriers against treatment. With the evidenced conditional probability matrix of regenerative medicine (a cancer immunotherapy drug), we can compute a corresponding predictive model from the approximated Bayesian regression. Hence, the efficiency of regenerative medicine can be calculated, which can then be evaluated alongside daily operation data for further improvement. Additionally, genes can be projected onto a planar surface, and thus a genetic mapping can be created. This can be developed from traditional mathematics: topology and complex geometry. I employ a step-by-step approach to the final result: a complex logarithmic function that maps to a planar surface. The mapping event can also be intuitively thought of as the genetic spiral helix to the planar (while I lack sufficient DNA data and cannot verify). The result is encouragement of the use of spatial analysis for population genetics for the purpose of personalized and regenerative medicine. This implies the individual patient will receive a tailor-made treatment that minimizes side effects. The beginning of regenerative medicine started from the ancient story of Theogony's theft of fire.

\footnotetext{
${ }^{20}$ https://www.frontiersin.org/articles/10.3389/fgene.2019.00013/full
} 
Fast-forward to now, this paper depicts a picture of how one can create a predictive model for regenerative medicine. Inspired by the past, I describe how both preventive and regenerative medicine can be applied in the field of cancer treatment, enabling cancer patients to receive the best treatment in the future.

\section{List of Abbreviations:}

CRISPR — clustered regularly interspaced short palindromic repeats

DNA — deoxyribonucleic acid

\section{References:}

[1] Dixmier, J. (2010). General topology. New York: Springer-Verlag.

[2] Flapan, E., \& Averett, M. (2017). Knots, molecules, and the universe: an introduction to topology. Telangana: American Mathematical Society.

[3] Lipschutz, S. (1987). Schaums outline of theory and problems of general topology. Singapore: McGraw-Hill.

[4] Pérez-Nimo, M.M \& Camúñez-Ruiz1, J.A. Matrix Form of The Bayes Theorem And Diagnostic Tests, IOSR Journal of Mathematics. Retrieved from http://www.iosrjournals.org/iosrjm/papers/Vol14-issue6/Version-1/A1406010106.pdf

[5] Polykandriotis, E., Popescu, L. M., \& Horch, R. E. (2010). Regenerative medicine: then and now - an update of recent history into future possibilities. Journal of Cellular and Molecular Medicine, 14(10), 2350-2358. doi: 10.1111/j.1582-4934.2010.01169.x 
[6] Runde, V. (2008). A taste of topology. New York: Springer.

[7] Shick, P. L. (2007). Topology: point-set and geometric. Hoboken: Wiley-Interscience.

[8] Viro, O. Y. (2008). Elementary topology: problem textbook. Providence, RI: American Mathematical Society.

[9] Waldmann, S. (2014). Topology: an introduction. London: Springer.

[10] Wilkins, D. R. (1988). Algebraic Topology. Retrieved from

https://www.maths.tcd.ie/ dwilkins/Courses/421/421Mich88.pdf.

[11] https://www.nature.com/articles/s41590-019-0588-6

[12] https://www.nature.com/articles/s41590-019-0578-8

[13] https://www.ncbi.nlm.nih.gov/pmc/articles/PMC6716904/

[14] https://www.ncbi.nlm.nih.gov/pmc/articles/PMC5930008/

[15] http://www.math.uchicago.edu/ may/VIGRE/VIGRE2011/REUPapers/Dooley.pdf

[16] https://ghr.nlm.nih.gov/primer/genomicresearch/genomeediting

[17] $\underline{\mathrm{ibid}}$

[18] https://www.analyticsvidhya.com/blog/2016/06/bayesian-statistics-beginners-simple-english/ 
[19] ibid

[20] http://pi.math.cornell.edu/ hatcher/AT/AT-doublepage.pdf

[21] https://www.nature.com/articles/s41590-019-0578-8

[22] https://www.youtube.com/watch?v=ERun58CeOsA

[23] Lam, K.S. (March 2020) Evaluation of the Weather-Influenza Pattern with a Regression Model Approximation to Causality Human Health \& Disease eJournal, Vol 4, Issue 69, June 03, 2020; Infectious Disease eJournal, Vol 2, Issue 57, June 03, 2020; Planetary Health eJournal, June 08,2020 retrieved from https://papers.ssrn.com/sol3/papers.cfm?abstract_id=3563601

[24] https://www.scmp.com/news/hong-kong/health-environment/article/3047956/china-coronavirus-hong-kong-researchers-have

[25] https://hub.hku.hk/cris/rp/rp02139

[26] https://www.physics.hku.hk/people/academic/347

[27] https://hkumath.hku.hk/ imr/IMRPreprintSeries/2011/IMR2011-10.pdf 


\section{Remarks:}

This author remarks that rather than modelling the conversion of cancer cells to normal cells, there have already been newly developed immune cell therapies for cancer treatment reported in England (January 2020). The theory is that one uses the genome-wise-CRISPR-Cas9 screening for the creation of a T-cell receptor (TCR). As a result, the TCR can recognise and kill nearly all human cancer types. Indeed, researchers in the Cardiff University discovered that most kinds of the cancer cells had the monomorphic MHC class I-related protein (MR1) where they can be realised by the TCR but remained stable to noncancerous cells ${ }^{21}$ [21]. This is a new method in T-cell immune therapy. In the coming years, I believe that scientists will further develop commonly used cancer healing drugs, possibly through genetic engineering human blood T-cells that could be used as an antigen to MR122 [22]). This would therefore be capable of detecting and destroying every type of cancer thanks to the special characteristics of MR1.

On the other hand, I suggest that one may apply a similar regression model technique (which will be shown in Lam, 2020 [22]) to approximate the (breast) cancer cell to normal (fat) cell conversation causality link according to different influencing conditions or factors. Professionals can then develop the most suitable therapy in order to facilitate and achieve the best conversion with reference to the corresponding particular approximation models.

In addition to the application in DNA 2D mapping, one may also use the same 2D mapping technique for aerodynamics in physics (or engineering). Indeed, we can design our future

\footnotetext{
${ }^{21}$ https://www.nature.com/articles/s41590-019-0578-8

${ }^{22}$ https://www.youtube.com/watch?v=ERun58CeOsA
} 
airplanes with reference to the 2D mapping of the spoiler-effect ${ }^{23}[23]$ such as the airplane's wing, etc. Then one may perform the corresponding spatial analysis ${ }^{24}[24]$ to a particular part and thus improve the upward lifting force for the whole airplane. Furthermore, from the projected 2D image, we can print the subsequent 3D model. One may then get an in-depth view of the investigating object's spoiler-effect. Hence, one may maximise the upward lifting force and minimise the downward force in a better and easier way. The result will encourage more efficient use of energy during the process of the aircraft taking off.

Another practical use of topology is in the application of condensed matter during fusion. Indeed, one may also apply the Bayesian analysis in computing the probability of quantum tunnelling. I note that the effect of quantum tunneling happens every day during the process of fusion from our main energy source - the sun. In other words, by studying these two pairs of subjects: "quantum tunnelling through Bayesian analysis" and the "topology with the condensed matter in fusion" - one can have an in-depth understanding of the fusion procedure. Hence, one can further develop our next generation clean energy source ${ }^{25}[25]$ - a "fusion electric plant".

\footnotetext{
23 https://www.scmp.com/news/hong-kong/health-environment/article/3047956/china-coronavirus-hong-kong-researchers-have

24 https://hub.hku.hk/cris/rp/rp02139

25 https://www.physics.hku.hk/people/academic/347
} 
Figures

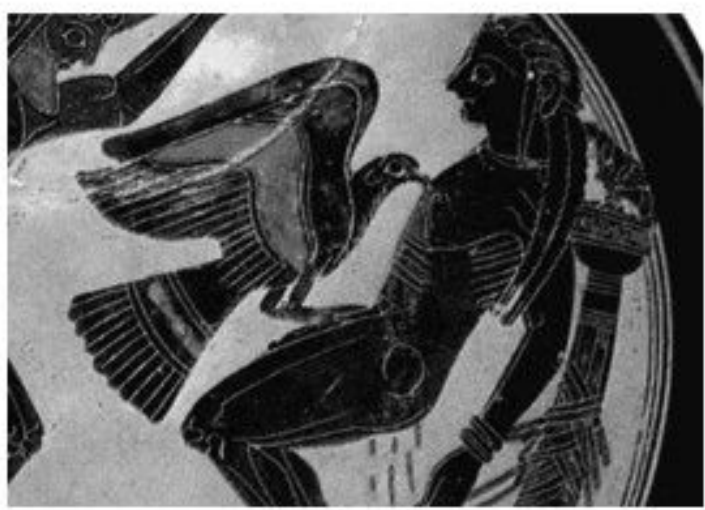

\section{Figure 1}

The myth of the titan Prometheus provides an icon

The complex logarithm function maps a Riemann surface to $R^{3}\{0\}$ as illustrated below (Shick, 2007) [7]:

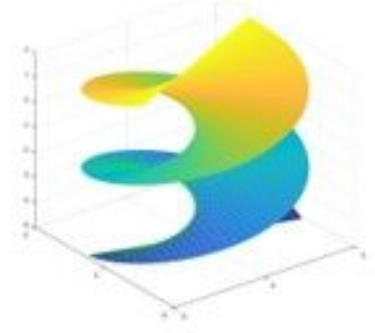

As the complex logar thmic function can

be deformed and mapped to a Riemann surface, hence the DNA spiral helix can also be divided and mapped in a similar way to a plame surface once the covering spaces condition is verified. The intuitive idea can be further developed for the $3 \mathrm{D}$ space reduction to $\mathrm{a} 2 \mathrm{D}$ plame space in gene mapping.

\section{Figure 2}

The complex logarithm function map 


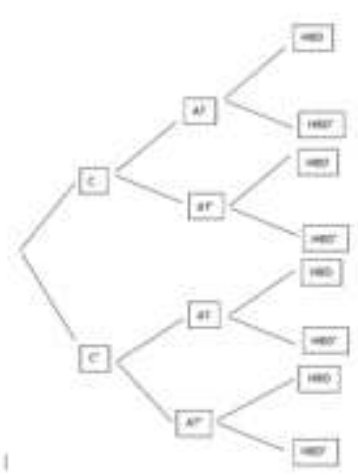

Key: $\mathrm{C}$ - cancer

AT - Attain treatment

Figure 3

Bayesian Tree Diagram 